\section{OP0061 A PROOF-OF-CONCEPT STUDY TO ASSESS THE EFFICACY OF TOCILIZUMAB MONOTHERAPY AFTER ULTRA-SHORT GLUCOCORTICOID ADMINISTRATION TO TREAT GIANT CELL ARTERITIS - THE GUSTO TRIAL}

L. Christ ${ }^{1}$, L. Seitz ${ }^{1}$, G. Scholz ${ }^{1}$, A. C. Sarbu ${ }^{1}$, J. Amsler ${ }^{1}$, L. Bütikofer ${ }^{2}$, C. Tappeiner ${ }^{3}$, F. Kollert ${ }^{1}$, S. Reichenbach ${ }^{1}$, P. Villiger ${ }^{1} .{ }^{1}$ Inselspital, Bern University Hospital, Department of Rheumatology, Immunology and Allergology, Bern, Switzerland; ${ }^{2}$ Inselspital, University of Bern, Clinical Trial Unit (CTU), Bern, Switzerland; ${ }^{3}$ Inselspital, Bern University Hospital, Department of Ophthalmology, Bern, Switzerland

Background: Two randomised controlled trials [1, 2] demonstrated a glucocorticoid (GC)-sparing effect of tocilizumab (TCZ) of at least $50 \%$. Long-term GC treatment leads invariably to numerous side effects, particularly in elderly giant cell arteritis (GCA) patients.

Objectives: The GUSTO (GCA treatment with ultra-short GC and TCZ) trial was set up to evaluate the efficacy and safety of TCZ-monotherapy after ultra-short GC treatment in new-onset GCA.

Methods: Eighteen patients with newly diagnosed GCA were enrolled in this investigator-initiated, single-arm, single-center, open-label clinical trial with Simon's two stage design (NCT03745586). Patients received $500 \mathrm{mg}$ methylprednisolone intravenously for 3 consecutive days. Thereafter, GC treatment was discontinued and TCZ (8 mg/kg body-weight) was administered intravenously, followed by weekly subcutaneous TCZ injections $(162 \mathrm{mg})$ from day 10 until week 52. The primary endpoint was defined as the proportion of patients who achieved remission within 31 days and showed no relapse at week 24; the secondary endpoint included the proportion of patients with complete relapse-free remission of disease at week 52. Remission was defined as disappearance of GCA symptoms, whereas partial remission included the presence of mild symptoms (defined as non-ischemic with NRS $<5 / 10$, reported as mild, not occurring on most days of the week). An interim analysis of the primary endpoint was performed after the first 12 patients reached the primary endpoint.

Results: At baseline there were 12/18 female patients, and the median age was 71 (range 64-78) years. Overall, 15/18 had cranial symptoms (10/18 had jaw claudication, 6/18 had visual symptoms), 10/18 suffered from polymyalgia rheumatica (PMR)-symptoms, 16/18 had positive cranial ultrasound, and 13/18 had positive histopathology.

At interim analysis, only $25 \%(3 / 12)$ of patients achieved remission within 31 days and stayed relapse-free up to week 24 . Thus, the null hypothesis that the proportion of responders would be smaller than $40 \%(p=0.92)$ was not rejected. Of the 18 patients recruited at the time of interim analysis, 14 achieved remission within 24 weeks (mean duration 11.1 (95\% Cl 8.3-13.9) weeks) and 13 showed no relapses up to 52 weeks $(72 \%, 95 \% \mathrm{Cl} 47-90 \%)$. Overall, $3 / 18$ patients were non-responders $(2 / 3$ with persistent cranial symptoms including one new-onset of an anterior ischemic optic neuropathy (AION); $1 / 3$ with persistent PMR symptoms) and started on rescue GC-treatment, and 2/18 discontinued the study due

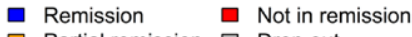

$\square$ Partial remission $\square$ Drop out

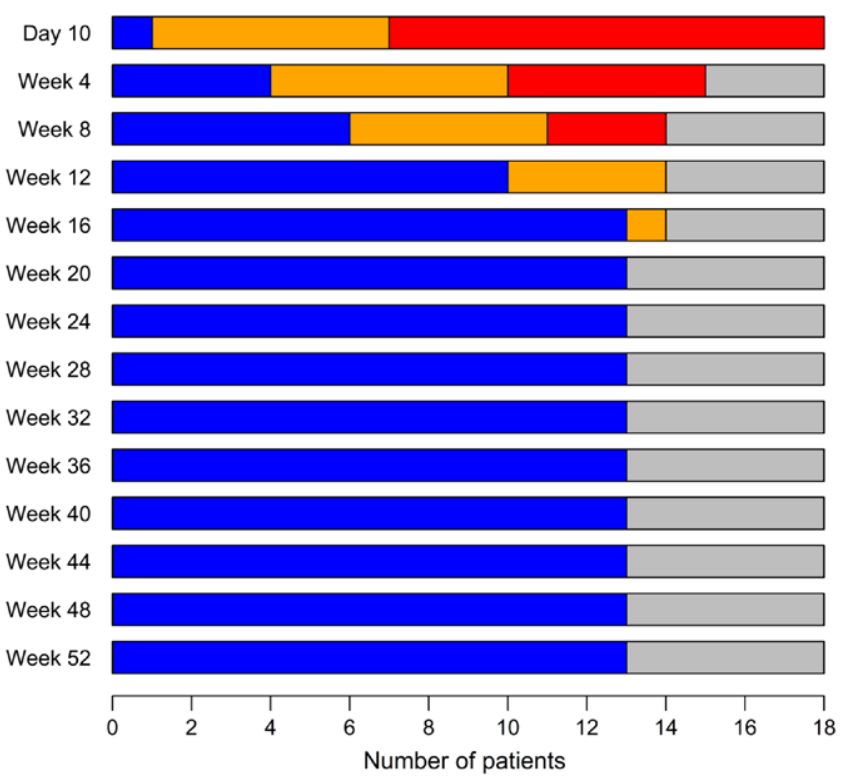

Figure 1. Disease status of patients at each visit (Day 10 - week $52, n=18$ ). to an adverse event (hepatopathy and diverticulitis, respectively; 1/2 after induction of remission).

Figure 1 demonstrates remission status over time.

Conclusion: After a 3-days pulse of methylprednisolone, ensuing TCZ monotherapy induced and maintained remission until week 52 in 13/18 patients. The data add an important piece of evidence regarding the potency of blocking the interleukin-6 pathway in GCA and suggest that a substantial reduction of concomitant GC treatment in TCZ-treated GCA patients is feasible.

\section{REFERENCES:}

[1] Villiger, P.M., et al., Tocilizumab for induction and maintenance of remission in giant cell arteritis: a phase 2, randomised, double-blind, placebo-controlled trial. Lancet, 2016. 387(10031): p. 1921-7.

[2] Stone, J.H., et al., Trial of Tocilizumab in Giant-Cell Arteritis. N Engl J Med, 2017. 377(4): p. 317-328.

Disclosure of Interests: Lisa Christ Shareholder of: F. Hoffmann-La Roche, Grant/research support from: Gilead Sciences; F. Hoffmann-La Roche; Pfizer, Luca Seitz: None declared, Godehard Scholz: None declared, Adela-Cristina Sarbu: None declared, Jennifer Amsler: None declared, Lukas Bütikofer: None declared, Christoph Tappeiner: None declared, Florian Kollert Shareholder of Roche, Consultant of: Yes (Actelion, BMS, Boehringer-Ingelheim, Pfizer), Grant/ research support from: Yes (Gilead, Pfizer), Employee of: Yes, I am currently employed by Roche and previously by Novartis, Stephan Reichenbach: None declared, Peter Villiger Speakers bureau: Roche, MSD, Abbvie, Pfizer, Novartis Grünenthal, Celgene, Sanofi, Chugai, Consultant of: Roche, MSD, Abbvie, Pfizer Novartis, Celgene, Sanofi, Grant/research support from: Roche, MSD, Abbvie DOI: 10.1136/annrheumdis-2021-eular.522

\section{Vasculitis - II}

\section{OP0062 CYTOKINE PRODUCING B CELLS SKEW MACROPHAGES TOWARDS A PRO-INFLAMMATORY PHENOTYPE IN GIANT CELL ARTERITIS}

J. C. Graver ${ }^{1}$, W. F. Jiemy ${ }^{1}$, D. Altulea ${ }^{1}$, A. Boots ${ }^{1}$, P. Heeringa ${ }^{2}$, W. Abdulahad ${ }^{1}$, E. Brouwer ${ }^{1}$, M. Sandovici ${ }^{1}{ }^{1}$ University of Groningen, University Medical Center Groningen, Rheumatology \& Clinical Immunology, Groningen, Netherlands; ${ }^{2}$ University of Groningen, University Medical Center Groningen, Pathology \& Medical Biology, Groningen, Netherlands

Background: Giant cell arteritis (GCA) is the most frequent form of systemic vasculitis affecting the temporal artery (TA) and the aorta. Macrophages and T cells are well recognized players in the pathogenesis of GCA while B cells are often not taken into account. Recently, changes in the circulating B cell compartment were documented in GCA and B cells were found to organize into tertiary lymphoid organs at the site of vascular inflammation (TA and aorta). ${ }^{1,2}$ The exact role of $B$ cells in GCA is still unknown as no disease-specific antibodies have been defined However, beside their role in humoral immunity, B cells can also produce various cytokines. In GCA, peripheral B cells of treated GCA patients, showed an enhanced capacity to produce pro-inflammatory cytokine Interleukin (IL) $6^{3}$ which is nowadays an important target of treatment in GCA. ${ }^{4}$ We hypothesize that B cells help shape the inflammatory response in GCA by producing effector and regulatory cytokines. Objectives: We aimed to assess the cytokine profile of circulating and lesional $B$ cells in GCA and studied the effects on macrophage skewing.

Methods: To assess B cells with the capacity to produce cytokines, cryopre served peripheral blood mononuclear cells of 11 untreated GCA patients and 15 age- and sex-matched healthy controls $(\mathrm{HC})$ were cultured for 3 days in the presence of CpG-ODN 2006. During the last 5 hours phorbol myristate acetate and Calcium lonophore were added. Thereafter, intracellular effector (IL6, TNFa, IFNy, LTb) and regulatory B cell-related cytokines (IL10) were measured with flow cytometry. To assess potential skewing of macrophages by $B$ cell products, THP-1 cells were differentiated into macrophages and stimulated for 24 hours with supernatant from stimulated B cells $(n=6)$. Expression of IL23, IL6, IL1b, TNFa, MMP9 and YKL40 was assessed on mRNA level with qPCR. To assess local cytokine production, TA $(n=11)$ and aorta tissue samples $(n=10)$ of histologically-proven GCA patients were stained to detect CD20, IL6, TNFa, IFNy, LTb, and IL10 expression. For comparison, 14 aorta tissues samples of patients with an atherosclerosis-related aortic aneurysm were included.

Results: In vitro stimulated B cells from untreated GCA patients showed an enhanced percentage of IL6+ B cells (median (IQR); 44 (41-52)) and of IL6+T$\mathrm{NFa}+\mathrm{B}$ cells (12 (8-24)) compared to stimulated B cells from HC (IL6+: 28 (2339), IL6+TNFa+: 6 (4-24)). In addition, soluble factors, secreted by GCA derived and stimulated $B$ cells, skewed macrophages towards a pro-inflammatory phenotype with enhanced expression of IL23, IL6, IL1b, and TNFa. Furthermore, these macrophages also showed higher expression of the tissue remodelling factor MMP9 and the pro-angiogenic factor YKL40. At the site of vascular inflammation, $B$ cells were detected in the regions with clear TNFa, IL6, IFNY, LTb and IL10 expression in both the TA and aorta of GCA patients. 